\title{
The Configurational Statistics of a Polymer Confined to a Wedge of Interior Angle $\alpha$
}

\author{
J. I. Lauritzen* and E. A. DiMarzio \\ National Measurement Laboratory, National Bureau of Standards, Washington, D.C. 20234
}

(April 3, 1978)

\begin{abstract}
The probability distribution for the end-to-end length of a polymer of $N$ segments confined in a wedge of interior angle $\alpha$ is obtained $(2 \pi \geq \alpha>0)$. The result is used to evaluate the partition function, $Q$, for the cases: (1) one end free-one end tied to the vertex, $Q$ is proportional to $\gamma^{N} N^{-\pi / 2 \alpha}$ both ends tied to each other at the vertex, $Q$, is proportional to $\gamma^{N} N^{-3 / 2-\pi / \alpha}$.
\end{abstract}

Key words: Polymer Statistics; polymer surface problem; polymer in a wedge; polymer interface; probability distribution of polymer near surface.

\section{Introduction}

It has been shown in previous work $[1,2]^{1}$ that the effects of a surface on the configurational statistics of a polymer can be obtained by solving the following equations:

$$
\begin{aligned}
\frac{\partial w}{\partial t} & =D \nabla^{2} w \\
w & =0 \text { on surface } \\
w(t=0) & =\delta\left(x-x_{0}, y-y_{0}, z-z_{0}\right)
\end{aligned}
$$

where

$$
D=n l^{2} / 6, t=N / n \text {. }
$$

and $\delta$ is the three-dimensional Dirac delta function. $w(x, y$, $z, t)$ is the probability density of a chain end being at $(x, y$, $z$ ) after $N$ steps, $n$ is the number of steps per unit time, and $l^{2}$ is the expected square of the length of an individual step. The above equation holds when the surface acts as a hard core barrier to the passage of the polymer segments. The case of long and/or short range attraction of the surface for the polymer segments is amenable to other techniques [3, 4] for some simple geometries.

The problem of the configurational freedom of a polymer near a surface is relevant to the problem of determining the manner of incorporation of a polymer molecular during the crystallization process. In fact, a result of this paper (eq (28)) has been used to derive an expression for the contribution to the surface free energy arising from cilia [5].

\footnotetext{
* Deceased.

${ }^{1}$ Figures in brackets indicate the literature references at the end of this paper.
}

\section{Theory}

Let us consider the specific surface geometry of a wedge defined by figure 1 . The polymer is attached to the point defined by $\rho=\rho_{0}, z=0, \phi=0$ in cylindrical coordinates.

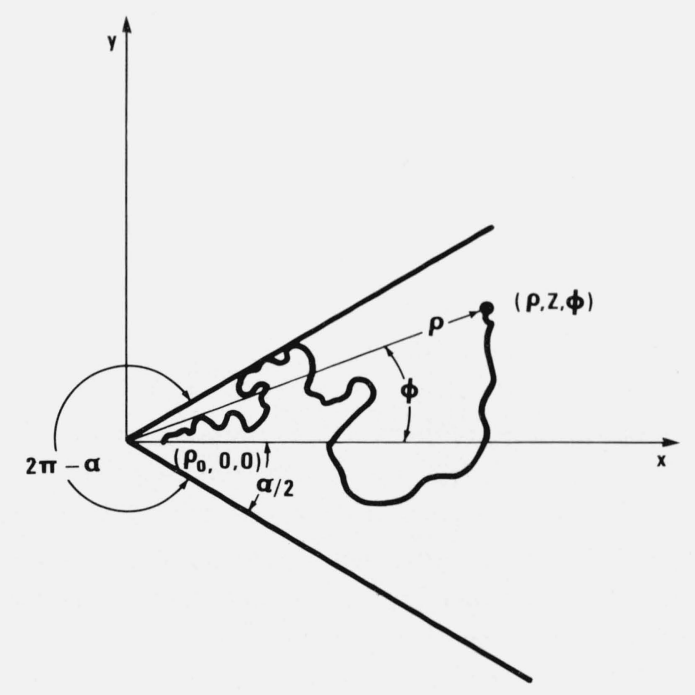

Figure 1. A polymer molecular confined to a wedge of angle $\alpha$ has fewer configurations than one not so confined.

The purpose of the paper is to calculate the effect of this confinement.

It is permitted to wander anywhere within the angle $\alpha$ of the wedge. The impenetrable cyrstal is imagined to occupy the interior of the angle $2 \pi-\alpha$. In the absence of the wedge the partition function would be 


$$
Q_{0}=\gamma^{N}
$$

where $\gamma$ is a partition function per bead. Since the boundary condition annihilates all walks that touch the boundary the partition function (sum over states) in the presence of the boundary is

$$
Q=\gamma^{N} \int w d v .
$$

where the integration extends over the volume of the wedge. This result is valid for large $N$ because the diffusion equation approach of eqs (1-2) is valid for large $N[1,2]$. If we let

$$
\tau=4 D t=\frac{2}{3} l^{2} N
$$

then the diffusion equation becomes

$$
4 \frac{\partial w}{\partial t}=\nabla^{2} w
$$

The complete statement of the problem, then, in cylindrical coordinates is

$$
\begin{aligned}
4 \frac{\partial w}{\partial \tau} & =\frac{1}{\rho} \frac{\partial}{\partial \rho}\left(\rho \frac{\partial w}{\partial \rho}\right)+\frac{\partial^{2} w}{\partial z^{2}}+\frac{1}{\rho^{2}} \frac{\partial^{2} w}{\partial \phi^{2}} \\
w & =0, \phi= \pm \frac{\alpha}{2} \text { for all } \rho, z, \tau \\
w & =0 \text { as } \rho \rightarrow \infty \text { and } / \text { or } z \rightarrow \infty \\
w(\tau=0) & =\delta\left(\rho-\rho_{0} z, \phi\right)
\end{aligned}
$$

where the delta function locates the source at $\left(\rho_{0}, 0,0\right)$.

The problem as formulated in eq (7) is identical to the problem of heat conduction in a wedge. Carslaw [6] has solved the problem in this context for arbitrary angle $\alpha$ and for a source point at any location. Accordingly, we could have quoted his general result and derived from it the more special result, eq (17), on which the remainder of the paper is based. However, such a procedure would not have resulted in appreciably fewer equations than the procedure of this paper. Also, our procedure is simpler than the generalized method of images used by Carslaw.

The Green's function of eq (7) in the absence of boundary conditions is

$$
\frac{1}{(\pi \tau)^{3 / 2}} \exp \left(-\frac{z^{2}}{\tau}-\frac{\left(\rho^{2}+\rho_{0}^{2}-2 \rho \rho_{0} \cos \phi\right)}{\tau}\right) .
$$

Our solution must approach the above expression as $\tau \rightarrow 0$ since in this limit both eq (8) and $w$ are delta functions.
We expect the dependence on $z$ of the solution, $w$, to be $\exp \left(-z^{2} / \tau\right)$. Without loss of generality we postulate

$w_{s}=\frac{1}{\tau^{3 / 2}} e^{\frac{-\left(\rho^{2}+\rho_{0}^{2}+z^{2}\right)}{\tau}} \cos \left(\frac{(2 s+1) \pi \phi}{\alpha}\right) F_{s}(\rho, \tau)$.

The $\cos \left((2 s+1) \frac{\pi \phi}{\alpha}\right)$ forces $w_{s}$ to be zero on the surface and any function of $\rho, \tau$, and $\phi$ which is zero at $\phi$ $= \pm \alpha / 2$ can be obviously represented by a sum of $w_{s}$ over $s$. The differential equation (eq (7)) becomes

$$
\begin{aligned}
4 \frac{\partial F_{s}}{\partial \tau}=\frac{\partial^{2} F_{s}}{\partial \rho^{2}}+\frac{1}{\rho} \frac{\partial F_{s}}{\partial \rho} & -\frac{4 \rho}{\tau} \frac{\partial F_{s}}{\partial \rho} \\
& -\left\{\frac{4 \rho_{0}^{2}}{\tau^{2}}+\frac{(2 s+1)^{2} \pi^{2}}{\alpha^{2} \rho^{2}}\right\} F_{s} .
\end{aligned}
$$

Thus, eq (9) satisfies both the differential equation and the boundary condition provided only that $F_{s}$ is chosen to satisfy eq (10). We now wish to solve this equation for $F_{s}$. It will simplify our problem if we transform the independent variables $\rho$ and $\tau$ to $m=2 \rho \rho_{0} / \tau$ and $\tau$.

$\frac{\partial^{2} F_{s}}{\partial m^{2}}+\frac{1}{m} \frac{\partial F_{s}}{\partial m}-\left\{1+\frac{(2 s+1)^{2} \pi^{2}}{\alpha^{2} m^{2}}\right\} F_{s}=\frac{\tau^{2}}{\rho_{0}^{2}} \frac{\partial F_{s}}{\partial \tau}$.

This equation can be solved by separation of variables. At this point we make the observation that if we can satisfy the initial condition by a sum of terms of the form of eq (9) over $s$, then we will have solved our problem. If $F_{s}$ depends only on $m$; i.e.; is not explicitly dependent on $\tau$ in eq (11), then a sum of terms of the form of eq (9) can be made to approach eq (8) in the limit of $\tau=0$, and the initial condition will be satisfied. Equation (11) then reduces to

$$
\frac{d^{2} F_{s}}{d m^{2}}+\frac{1}{m} \frac{d F_{s}}{d m}-\left\{1+\frac{(2 s+1)^{2} \pi^{2}}{\alpha^{2} m^{2}}\right\} F_{s}=0 .
$$

We write the solution of eq (12) as

$$
F_{s}=A I_{(2 s+1) \frac{\pi}{\alpha}}(m)+B K_{(2 s+1) \frac{\pi}{\alpha}}(m)
$$

where $I$ and $K$ are the modified Bessel Functions of the first and second kind. Now as $\rho \rightarrow 0$, and then $K_{(2 s+1) \frac{\pi}{\alpha}}(m)$ diverges. Since $F_{s}$ must vanish as $\rho \rightarrow 0$ for $|\phi| \leq \alpha / 2$ it follows that $B$ must be set equal to zero. Then our expression for $w$ becomes

$$
\begin{aligned}
w=\frac{1}{\tau^{3 / 2}} e^{-\frac{\left(\rho^{2}+\rho_{0}^{2}+z^{2}\right)}{\tau} \sum_{s=0}^{\infty}} A_{s} \cos (2 s & \\
& +1) \frac{\pi \phi}{\alpha} I_{(2 s+1) \frac{\pi}{\alpha}}\left(\frac{2 \rho \rho_{0}}{\tau}\right) .
\end{aligned}
$$


We now must evaluate $A_{s}$. To do this we apply the requirement that eq (14) satisfy the initial condition.

$$
\lim _{\tau \rightarrow 0}\left\{w-\frac{1}{(\pi \tau)^{3 / 2}} e^{-\frac{z^{2}}{\tau}} e^{-\frac{\left(\rho^{2}+\rho_{0}^{2}-2 \rho \rho_{0} \cos \phi\right)}{\tau}}\right\}=0 .
$$

We multiply both' $w$ and $\frac{1}{(\pi \tau)^{3 / 2}} e^{-\frac{\left|\rho-\rho_{0}\right|^{2}}{\tau}}$ by $\cos (2 s+$ 1) $\frac{\pi \phi}{\alpha}$ and integrate $\phi$ from $-\alpha / 2$ to $\alpha / 2$ for some small but finite $\tau$. Then we pass to the limit of $\tau \rightarrow 0$. We find

$A_{s} \frac{\alpha}{2}=\lim _{\tau \rightarrow 0} \frac{1}{\tau^{3 / 2}} \int_{-\alpha / 2}^{\alpha / 2} \mathrm{~d} \phi \cos \frac{\tau \phi}{\alpha}(2 s$

$$
+1) \frac{e^{\frac{2 \rho \rho_{0} \cos \phi}{\tau}}}{\mathrm{I}_{(2 \mathrm{~s}+1) \frac{\pi}{\alpha}\left(\frac{2 \rho \rho_{0}}{\tau}\right)}}=\frac{2}{\sqrt{ } \cdot}
$$

This result was obtained by using asymptotic forms for $I$ and $\exp \left(\frac{2 \rho \rho_{0} \cos \phi}{\tau}\right)$. Equation (14) becomes

$$
\begin{aligned}
& w=\frac{4 \pi}{\alpha(\pi \tau)^{3 / 2}} \sum_{s=0}^{\infty} e^{-\frac{\left(\rho^{2}+\rho_{o}{ }^{2}+\mathrm{z}^{2}\right)}{\tau}} \cos (2 s \\
&+1) \frac{\pi \phi}{\alpha} I_{(2 s+1)} \frac{\pi}{\alpha}\left(\frac{2 \rho \rho_{0}}{\tau}\right) .
\end{aligned}
$$

When we evaluate these series terms for arbitrarily high $s$, we find that the series converges absolutely for any fixed finite $\rho, \phi$, and $\tau$. Equation (17), then, is the required solution since it satisfies the initial condition the boundary condition and the diffusion equation.

We now will evaluate

$$
W=\int_{o}^{\infty} \rho d \rho \int_{-\infty}^{\infty} d z \int_{-\alpha / 2}^{\alpha / 2} d \phi w
$$

where we have from eq (4)

$$
Q=\gamma^{N} W
$$

Integrating over $z$ and $\phi$ we find

$$
W=\frac{8}{\pi} \sum_{s=0}^{\infty} \frac{(-1)^{s}}{2 s+1} \int_{o}^{\infty} y d y e^{-y^{2}-a^{2}} I_{(2 s+1)} \frac{\pi}{\alpha}(2 y a)
$$

where

$$
a=\frac{\rho_{0}}{\sqrt{ } \tau} \text {, and } y=\rho / \sqrt{\tau} \text {. }
$$

We can express the integral over $y$ in terms of confluent hypergeometric functions. For example, if we let $t=y^{2}$, the integral is a Laplace transform, and from Tables of Integral Transforms [7], Vol. 1, p. 197 we have

$W=\frac{4}{\pi a} \sum_{s=0}^{\infty} \frac{(-1)^{s}}{2 s+1}$

$$
\frac{\Gamma\left(1+(2 s+1) \frac{\pi}{2 \alpha}\right)}{\Gamma\left(1+(2 s+1) \frac{\pi}{\alpha}\right)} e^{-\frac{a^{2}}{2} M_{-1 / 2,(2 s+1) \frac{\pi}{2 \alpha}}\left(a^{2}\right)}
$$

where $M_{-1 / 2,(2 s+1) \frac{\pi}{2 \alpha}}\left(a^{2}\right)$ is Whittaker's Function in the notation of the Bateman Project. This transforms to

$$
\begin{aligned}
& W=\frac{4}{\pi} e^{-a 2} \sum_{s=0}^{\infty} \frac{(-1)^{s}}{(2 s+1)} \frac{\Gamma\left(1+(2 s+1) \frac{\pi}{2 \alpha}\right)}{\Gamma\left(1+(2 s+1) \frac{\pi}{\alpha}\right)} \\
& \cdot a^{(2+1) \frac{\pi}{2 \alpha}} \Phi\left(1+(2 s+1) \frac{\pi}{2 \alpha},\right. \\
& \left.1+(2 s+1) \frac{\pi}{\alpha}, a^{2}\right) .
\end{aligned}
$$

where $\Phi(a, c, z)$ is Humbert's symbol for ${ }_{1} F_{1}(a, c, z)$ which is a generalized hypergeometric function.

First if we let $a \rightarrow \infty$, we have

$$
\lim _{a \rightarrow \infty} W=\frac{4}{\pi} \sum_{s=0}^{\infty} \frac{(-1)^{s}}{2 s+1}=1
$$

by the asymptotic expression given for $\Phi$ in the NBS Handbook of Mathematical Functions ${ }^{[8]}$. This shows that our expression is properly normalized.

We wish to evaluate $W$ for large $N$ (small $a$ ). For this purpose use the definiton of $\Phi={ }_{1} F_{1}$ given on page 373 of reference [7] to obtain

$$
\begin{aligned}
& \Gamma\left(1+(2 s+1) \frac{\pi}{2 \alpha}\right) \\
& \overline{\Gamma\left(1+(2 s+1) \frac{\pi}{\alpha}\right)} \\
& \left(1+(2 s+1) \frac{\pi}{2 \alpha}, 1+(2 s+1) \frac{\pi}{\alpha}, a^{2}\right)
\end{aligned}
$$




$$
=\sum_{k=0}^{\infty} \frac{\Gamma\left(k+1+(2 s+1) \frac{\pi}{2 \alpha}\right)}{\Gamma\left(k+1+(2 s+1) \frac{\pi}{\alpha}\right)} \frac{a^{2 k}}{k !} .
$$

$$
\frac{\Gamma\left(k+1+(2 s+1) \frac{\pi}{2 \alpha}\right)}{\Gamma\left(k+1+(2 s+1) \frac{\pi}{\alpha}\right)} \frac{a^{2 k+(2 s+1) \frac{\pi}{\alpha}}}{k !} .
$$

Equations (5) and (21) give

$$
a=\frac{\rho_{0}}{l} \sqrt{\frac{3}{2 N}} .
$$

We will let $\rho_{0} / l=1$ which means that the polymer is pinned one step away from the vertex.

We shall concern ourselves with the case $N$ large and shall calculate the first two terms in the expansion in powers of $a$. The leading term $(s=k=0)$ gives

$$
\frac{Q}{\gamma N}=\frac{4}{\pi} \frac{\Gamma\left(1+\frac{\pi}{2 \alpha}\right)}{\Gamma\left(1+\frac{\pi}{\alpha}\right)} a^{\pi / \alpha}=\frac{2 \Gamma\left(\frac{\pi}{2 \alpha}\right)}{\pi \Gamma\left(\frac{\pi}{\alpha}\right)}\left(\frac{3}{2 N}\right)^{\frac{\pi}{2 \alpha}} .
$$

In considering the first correction term, we have two cases:

First $2 \pi \geq \cdot \alpha>\pi$

$$
\begin{aligned}
& Q=\gamma^{N} \frac{2}{\pi}\left\{\frac{\Gamma\left(\frac{\pi}{2 \alpha}\right)}{\Gamma\left(\frac{\pi}{\alpha}\right)}\left(\frac{3}{2 N}\right)^{\frac{\pi}{2 \alpha}}\right. \\
& \left.-\frac{1}{3} \frac{\Gamma\left(\frac{3 \pi}{2 \alpha}\right)}{\Gamma\left(\frac{3 \pi}{\alpha}\right)}\left(\frac{3}{2 N}\right)^{\frac{3 \pi}{2 \alpha}} \cdots\right\}
\end{aligned}
$$

Second, when $\pi \geq \alpha>0$
$Q=\gamma^{N} \frac{2}{\pi} \frac{\Gamma\left(\frac{\pi}{2 \alpha}\right)}{\Gamma\left(\frac{\pi}{\alpha}\right)}$

$$
\left\{\left(\frac{3}{2 N}\right)^{\frac{\pi}{2 \alpha}}-\frac{\pi / 2 \alpha}{1+\pi / 2 \alpha}\left(\frac{3}{2 N}\right)^{\frac{\pi}{2 \alpha}+2} \ldots\right\}
$$

Notice that for small $a$, eq (26) is rapidly convergent.

\section{Discussion}

The main results of this paper are given by eq (17) for the probability, $w$, of a chain located at $(\rho, z, \phi)$ given that it started at $\left(\rho_{o}, 0,0\right)$ and eq (26) for the contribution to the partition function (eq (19)) due to the confining effects of the walls of the wedge.

Equation (28) proves a conjecture [9] made previously on the basis of knowledge of the results for $\alpha=0, \pi / 2$, and $\pi$.

Equation (17) can also be used to obtain the partition function when the terminal end is fixed at a given place. If we force it to the original starting point $\left(\rho_{o}, 0,0\right)$ we obtain by use of the limiting form of the modified Bessel function for small arguments.

$$
Q \propto \gamma^{N} / N^{\frac{3}{2}+\frac{\pi}{\alpha}}
$$

where $N$, which is proportional to $\tau$, is large.

Sincere thanks are due J. D. Hoffman for his helpful discussions and his continued interest in the problem, and to R. J. Rubin for a helpful suggestion.

\section{References}

[1] Pouchly, J., Collection Czeckoslov. Chem. Commun. 28, 1804 (1963).

[2] DiMarzio, E. A., J. Chem. Phys. 42, 2101 (1965).

[3] DiMarzio, E. A., and Rubin, R. J., J. Chem. Phys. 55, 4318 (1971), and references therein discuss the problem of plane boundaries.

[4] DiMarzio, E. A., and Guttman, C. M., J. Research NBS (submitted) and references therein discuss the Wiener integral techniques for a polymer immersed in a potential field.

[5] Hoffman, J. D., Frolen, L. J., Ross, G. S., and Lauritzen, J. I., Jr., J. Res. Nat. Bur. Stand. (U.S.) 79A (Phys. and Chem.) No. 6, 671-699 (Nov.-Dec. 1975)

[6] Carslaw, H. S., Conduction of Heat in Solids (Dover, New York, 1945), 2nd Edition. 
[7] Bateman, H., Bateman Manuscript Project, Tables of Integral Transforms, Volume 1 (McGraw-Hill, 1954).

[8] Abramowitz, M., and Stegun, I. A., Handbook of Mathematical
Functions (NBS Applied Math. Series. 55) (1964).

[9] DiMarzio, E. A., XXIII International Congress of Pure and Applied Chemistry, 8, 239 (1971). 